\title{
Preparation and in vivo anti-tumor evaluation of human papillomavirus E7 adjuvanted with Montanide ISA 266 as a vaccine candidate
}

\author{
Mashhadi Abolghasem Shirazi $\mathbf{M}^{1}$, Roohvand $\mathbf{F}^{2}$, Arashkia $\mathrm{A}^{2^{*}}$ \\ ${ }^{1}$ Department of Microbiology, Pharmaceutical Science Branch, Islamic Azad University, Tehran, Iran. \\ ${ }^{2}$ Department of Molecular Virology, Pasteur Institute of Iran, Tehran, Iran.
}

\begin{abstract}
Introduction: Human papillomavirus (HPV) 16 E7 protein is expressed constitutively by HPV-infected tumor cells. Mutant versions of E7 are considered as safer candidates for immunotherapy of cervical cancer. Different strategies including formulation with adjuvants are used to induce a potent immune response against antigenic proteins. Methods: In this experimental study, we used Escherichia coli as a host to recombinantly express wild-type E7 and its mutant non-oncogenic form as E7GGG. We formulated both antigens with Montanide ISA 266 adjuvant and evaluated IFN- $\gamma$ and IL-4 cytokines and antibody levels and also tumor regression in tumor-harboring C57BL/6 mice. Results: It was demonstrated that formulation of E7 and E7GGG antigens with Montanide ISA 266 resulted in a Th2-biased immune response. In the therapeutic mouse model, these formulations resulted in significant tumor regression compared to the control group. Conclusion: The formulation of the wild-type E7 and mutant E7GGG with Montanide ISA 266 might not be an optimal approach to regress TC-1 induced tumor; however, such combinations might be considered as an additive approach for stimulating the immune responses.
\end{abstract}

KEYWORDS: Human Papillomavirus 16, E7 Oncogene, Protein Vaccine.

\section{INTRODUCTION}

Cervical cancer is the second most common cancer amongst women worldwide. Persistent infection with human papillomavirus (HPV) is the main cause of cervical cancer; thus, prophylaxis and treatment of HPV infection is in high demand. Among approximately 15 different types of the cancer-causing HPV, $50 \%$ of them are caused by genotype 16 [1]. Existing licensed vaccines against HPV which are against L1 protein (late expressed major capsid protein) can effectively protect women from precursor lesions of cervical carcinoma caused by the most prevalent high-risk genotypes of HPV and most genital warts [1]. However, the therapeutical effects of these vaccines on the pre-existing HPV infection or premalignant lesions have not been demonstrated [2]. Therefore, there is an urgent need for developing new therapeutic vaccines against HPV.

In order to design an anti-HPV therapeutic vaccine, it is necessary to choose the proper target antigens with utmost care. It has previously been shown that early expressed proteins of HPV such as E6 and E7 are potential target candidates since they are viral proteins which are expressed constitutively by HPV-infected tumor cells and are also necessary for induction and maintenance of cellular transformation in the infected cells

*Corresponding Author: Arash Arashkia, Department of Molecular Virology, Pasteur Institute of Iran, Tehran, Iran.

Email: a_arashkia@pasteur.ac.ir

Tel/Fax: (+98) 2164112251
[3]. Different strategies have been developed to generate therapeutic vaccines against HPV-infected cells and these approaches are reviewed elsewhere [3-5]. Briefly, these approaches include using DNA [6], proteins [7, 8], peptides [9], live vectors [10, 11], and dendritic cells [12]. In patients infected with HPV type 16, cell-mediated immunity against E7 protein has been detected. Since E7 is one the oncogenic proteins of the virus, its mutant forms have been developed that have reduced carcinogenicity. One form of non-carcinogen E7 has point mutations in its amino acid residues 21,24 and 26 [13].

Protein-based vaccines are relatively safe and easy-to-produce candidates for developing a therapeutic vaccine. However, they need to be formulated by an adjuvant due to their deficiency in inducing a potent immune response [14]. Different forms of HPV oncogenic proteins have been used, formulated with different adjuvants such as QuilA [15], carrageenan, and CpG [16]. Montanide ISA (Seppic, France) is a group of ready-touse oil adjuvants that can be used for formulating different types of parenteral emulsions with immunostimulatory effects [17]. Montanide ISA 266 consists of a mixture of mineral oil and $50 \%$ metabolizable oil, as well as a highly refined surfactant obtained from mannitol and purified oleic acid [18]. In the present study, we expressed HPV16 wild-type E7 protein and a mutant non-oncogenic E7 (E7GGG) protein in a bacterial host and evaluated their effects along with Montanide ISA-266 
adjuant on the immune responses using an animal tumor model, in order to formulate a potent therapeutic vaccine candidate.

\section{MATERIALS and METHODS}

\section{Expression and purification of HPV16 E7 and E7GGG proteins}

HPV16 E7 coding sequence and the optimized E7GGG sequence were kindly provided by Dr. Azam Bolhasani and Dr. Mehdi Mahdavi (Pasteur Institute of Iran), respectively. The sequences were subcloned into pET-28a (+) vector (Novagen, USA) and transformed into Escherichia coli BL21 (DE3) host after being confirmed by PCR and restriction enzyme digestion. The protein expression was induced by adding $0.5 \mathrm{mM}$ IPTG at $\mathrm{OD}_{600}$ of $0.6-0.8$ at $37^{\circ} \mathrm{C}$ for $4 \mathrm{~h}$. The expression was analyzed by SDS-PAGE and Western blotting using His-probe Antibody (H-3) (Santa Cruz Biotechnology, USA). The expressed proteins were purified by His-tag using Ni/NTA column under denaturing condition. Finally, the purified proteins were dialyzed against phosphate-buffer saline (PBS; dialysis membrane $12 \mathrm{kDa}$ cut-off) and stored at $-70^{\circ} \mathrm{C}$.

The concentrations of the proteins were determined by Bradford assay [19].

\section{Antigen formulation and mice immunization}

The antigens were prepared by mixing $50 \mu \mathrm{g}$ purified protein with $20 \mu \mathrm{g}$ Montanide ISA 266. The mixture was dissolved in PBS at the final volume of $200 \mu \mathrm{l}$ under the sterile conditions.

Six to eight-week-old female C57BL/6 mice were purchased from Pasteur Institute of Iran (Karaj, Iran). The mice were housed for 1 week before the experiments, given free access to food and water. The mice were kept according to the animal ethics regulations of Pasteur Institute of Iran. In order to generate tumor tissue in the mice groups, one mouse was injected subcutaneously by TC-1 cell line which results in tumor model expression of HPV type 16 E6 and E7 proteins. After 1 month of tumor growth, the mouse was euthanized and the tumor was removed and cut into $2 \mathrm{~mm}^{3}$ fragments and each mouse of the test and the control groups received a fragment, subcutaneously. Test mice $(n=9)$ received either E7+Montanide 266 or E7GGG+Montanide 266 and the control mice received PBS. Mice were injected subcutaneously on days 0,14 , and 28 . Two weeks after the final injection, orbital sinus blood samples were collected. The mice were euthanized by cervical dislocation and the spleens were removed for further analyses.

\section{Cytokine assays}

Two weeks after the third immunization, a total number of $3 \times$ $10^{6}$ spleen cells/well were seeded in 24-well plate in RPMI 1640 and stimulated in vitro by adding $10 \mu \mathrm{g} / \mathrm{ml}$ of the protein antigen and incubated at $37^{\circ} \mathrm{C}$ in $5 \% \mathrm{CO}_{2}$, for 3 days. Supernatants were removed thereafter and the concentrations of murine IFN- $\gamma$ and IL- 4 cytokines were assessed by ELISA kits (Mabtech, Sweden) according to the manufacturer's instruction. The concentration of the cytokines in each sample was calculated according to the standard curve and the absolute cytokine concentration for each mouse was determined.

\section{Antibody isotyping}

Specific antibodies were determined by an optimized indirect ELISA. Briefly, $100 \mu \mathrm{l}$ of $10 \mu \mathrm{g} / \mathrm{ml}$ of the antigen in PBS was added into 96-well ELISA Maxisorp plates (Nunc, USA) and incubated overnight at $4^{\circ} \mathrm{C}$. The wells were washed with PBS containing $0.05 \%$ Tween 20 (PBS-T) and blocked for $1 \mathrm{~h}$ at $37^{\circ} \mathrm{C}$ with $5 \%$ skimmed milk in PBS (blocking buffer). The plates were washed with PBS-T and $100 \mu$ of $1: 100$ to $1: 12800$ diluted sera were added to each well and incubated at $37^{\circ} \mathrm{C}$ for $90 \mathrm{~min}$. The wells were washed with PBS-T and incubated for 90 min with HRP-conjugated anti-mouse Ab (Sigma, USA). The wells were then washed and incubated for $30 \mathrm{~min}$ with TMB substrate in the dark and the reaction was stopped with 2 $\mathrm{N}_{2} \mathrm{SO}_{4}$ and color density was measured at $\mathrm{OD}_{450} \mathrm{~nm}$. Detection of specific $\mathrm{IgG} 1$ and $\mathrm{IgG} 2 \mathrm{a}$ subclasses were carried out using goat anti-mouse IgG1 and IgG2a secondary antibodies (Sigma, USA), according to the manufacturer's instruction.

\section{Tumor growth inhibition assay}

As a therapeutic tumor model, the mice were implanted with the tumor and received antigen formulations or PBS three days after the implantation. The tumor size was measured every 3 days. The tumor diameters were measured in two orthogonal dimensions using electronic digital calipers. Tumor volumes were calculated as $\left(\right.$ length $\times$ width $\left.{ }^{2}\right) / 2$.

Statistical analysis

GraphPad Prism 7.0 (GraphPad Inc. USA) was used for statistical analysis. One-way ANOVA was performed to determine the differences of the immune responses among the various immunization groups. The statistical significance in tumor volumes was analyzed using Mann-Whitney U test. The data were expressed as means \pm SEM. Differences were considered statistically significant for $p<0.05$.

\section{RESULTS}

\section{Proteins expression and characterization}

The recombinant HPV16 E7 and E7GGG proteins were expressed in BL21 (DE3) strain and purified on Ni/NTA column. Sharp bands for E7 and E7GGG (19 and $17 \mathrm{kDa}$, respectively) were observed on $12 \%$ acrylamide gel. The results were confirmed by Western blotting using anti-His Ab (Fig. 1).

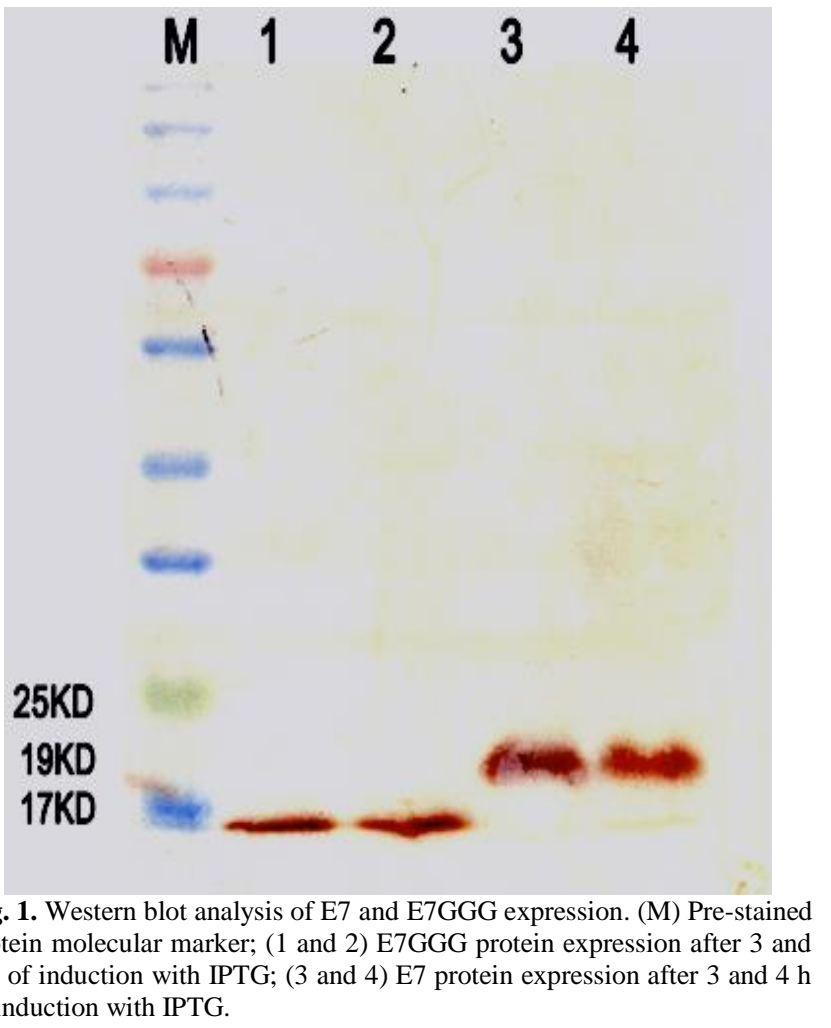
of induction with IPTG. 


\section{Secretion of IFN- $\gamma$ and IL-4 cytokines}

To determine the secretion levels of the cytokine after immunization of the tumor-bearing mice, IFN- $\gamma$ and IL-4 cytokines were measured from the isolated spleen cells. The results showed that mice immunized with E7+Montanide had slightly lower IFN- $\gamma$ (Fig. 2A) and higher IL-4 (Fig. 2B), compared with the mice immunized with E7GGG+Montanide. There were significant differences between the test and the control groups $(p<0.05)$.

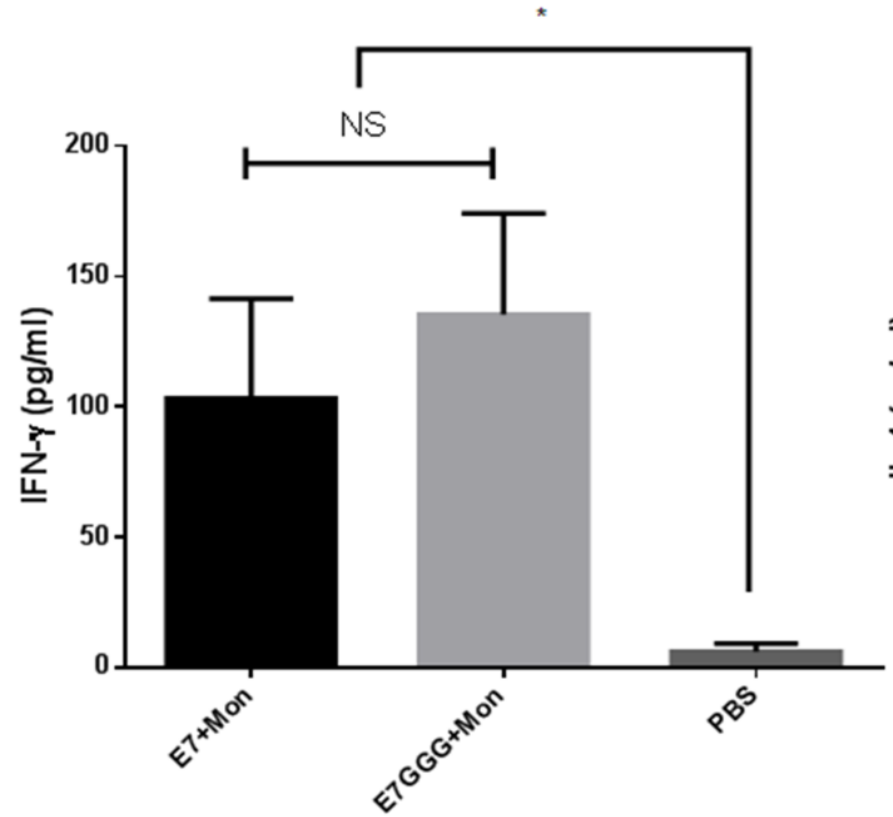

\section{Antibody Response}

Total IgG was evaluated by an indirect ELISA. In general, our results showed that Montanide 266 induced slightly more antibody response when combined with E7GGG compared to E7 protein (Fig. 3A). Evaluation of antibody isotyping test showed that there was no significant difference between E7 and E7GGG proteins formulated with Montanide 266, although there is slight increase in $\mathrm{IgG} 2 \mathrm{a}$ level in the E7GGG injected mice (Fig. 3B).

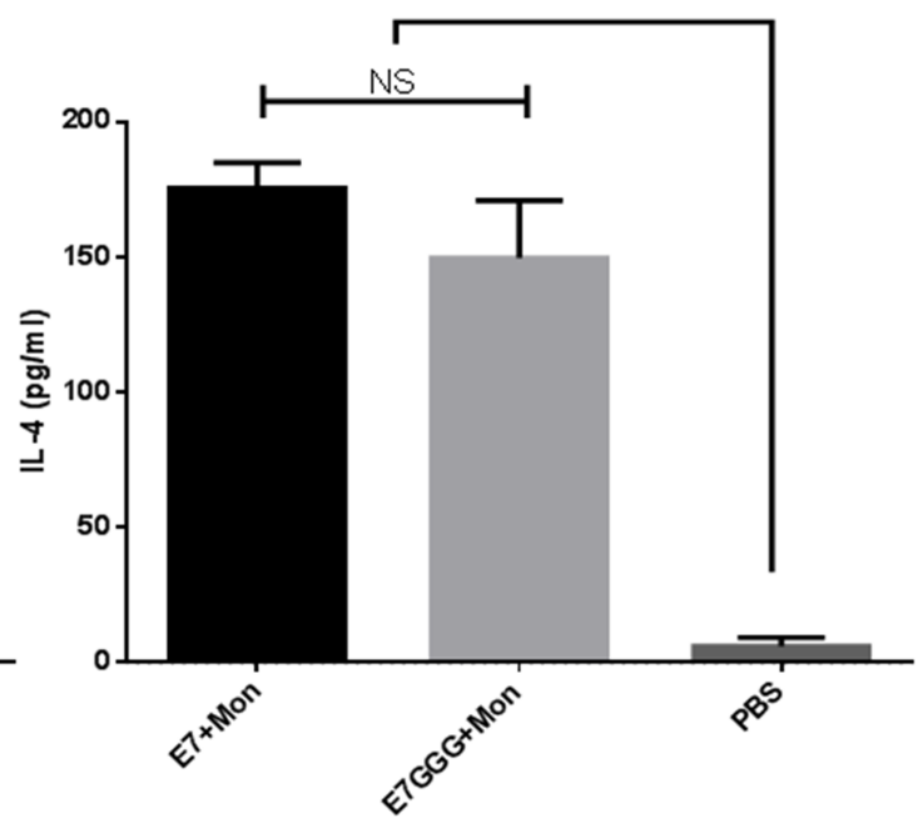

Fig. 2. Cytokines secretion analysis. A) IFN- $\gamma$ secretion by the isolated and cultured spleen cells in E7 and E7GGG immunized mice formulated in Montanide 266. B) IL-4 secretion by the isolated spleen cells in E7 and E7GGG immunized mice formulated in Montanide 266. [NS = no significant difference; Mon = Montanide 266].
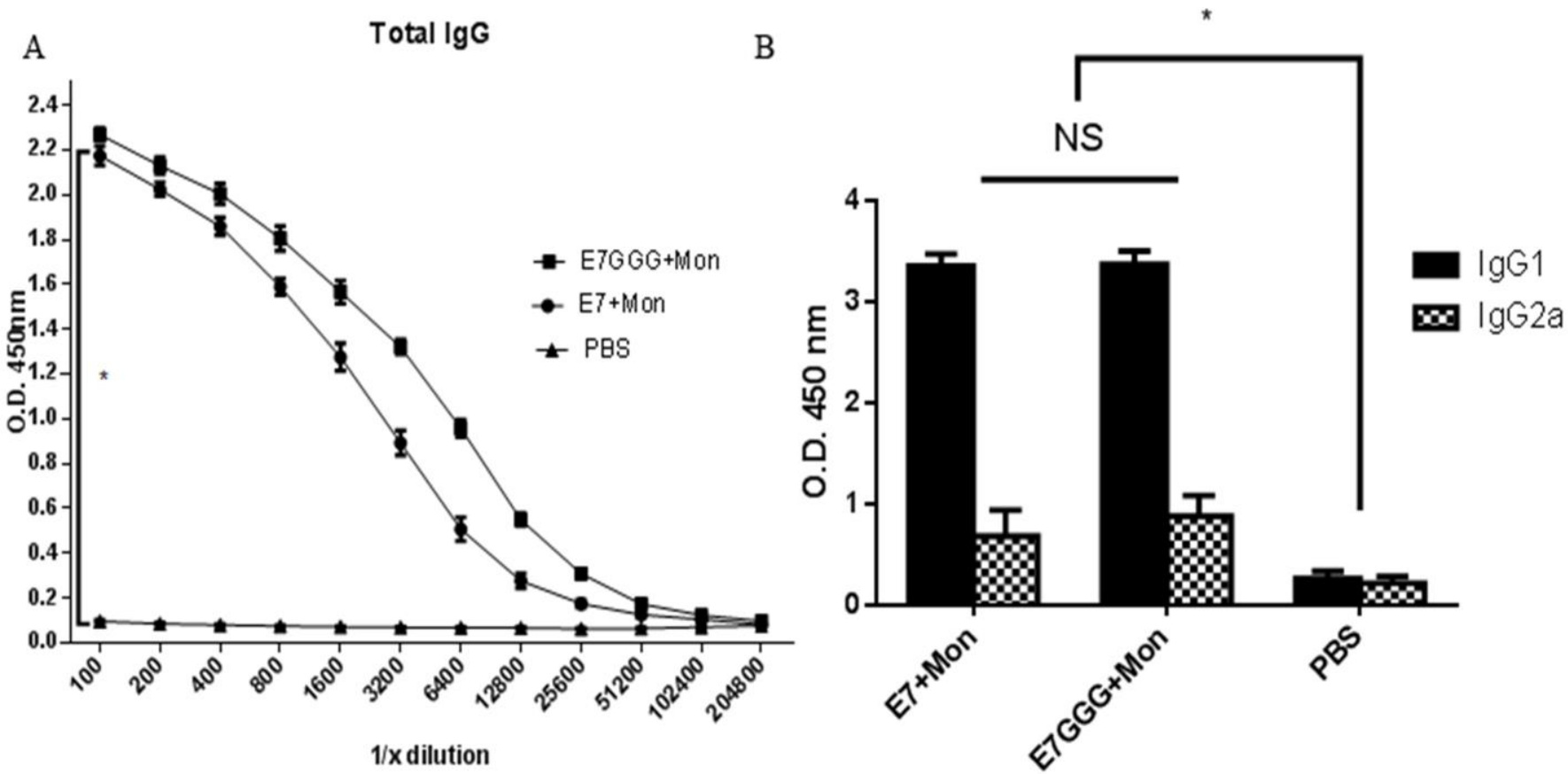

Fig. 3. Total and isotype antibody response. A) Total IgG titers in mice serum immunized either with E7 or E7GGG with Montanide 266. B) IgG1 and IgG2a levels in the immunized mice. [NS = no significant difference; Mon = Montanide 266] 


\section{Tumor regression assay}

Mice were implanted with TC-1 tumor cells. The tumor size was measured before immunization up to 24 days after the immunization. The results showed that mice injected with either E7 or E7GGG formulated with Montanide 266 could significantly reduce their tumor growth as compared to control group (Fig. 4) $(p<0.05)$.

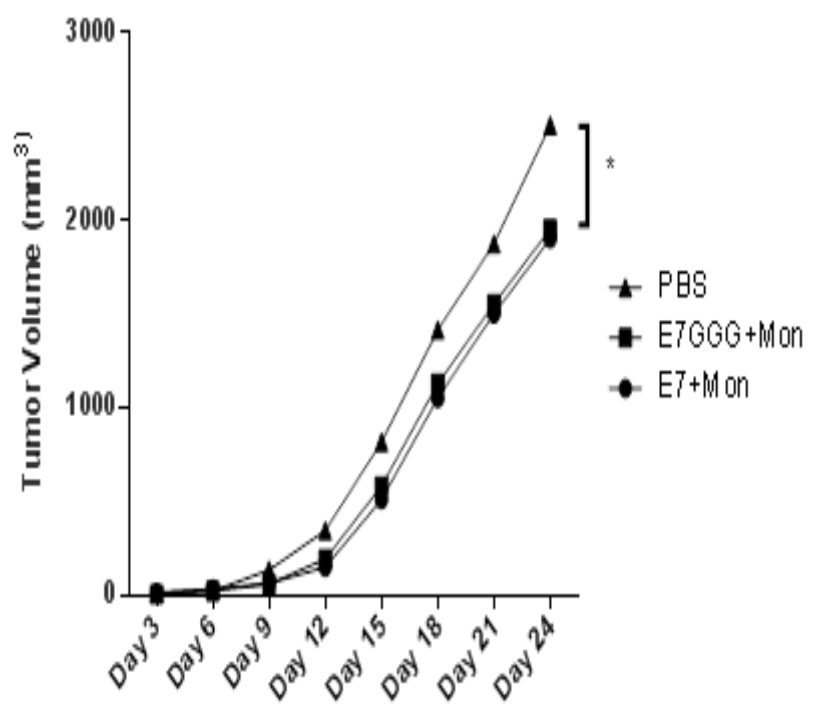

Days after Tumor Inoculation

Fig. 4. Tumor growth in the immunized and the control groups. Tumor size was measured for 3 weeks after the immunization with either E7+Mon or E7GGG+Mon [Mon = Montanide 266].

\section{DISCUSSION}

In the present study, we evaluated the immunogenicities of HPV-16 E7 and E7GGG proteins combined with Montanide ISA 266 as an adjuvant. Our results showed that these combinations induced Th2-skewed immunologic responses. Vaccination with exogenous whole proteins mainly induces Th2-related responses while E7 protein has been reported to evoke CD8 $\mathrm{T}$ cell responses when formulated with QuilA or CpG oligonucleotides [20, 21] . Between E6 and E7 oncogenic proteins, E7 has been previously reported to elicit stronger immunological protection compared with E6 protein [22]. Moreover, mutations in E7 reduce or completely abolish the transforming ability of the protein [23]. The mutated form of E7 protein used in this study was based on a previously published form, harboring D21G, C24G and E26G mutations [24]. Both E7 and E7GGG genes were expressed in E. coli and although their products were estimated to be approximately $11 \mathrm{kDa}$, they appeared as $17-19 \mathrm{kDa}$ on the polyacrylamide gel. Considering that the N-terminal domain of E7 protein (residues 1-38) consists of hydrophilic amino acids whereas the C-terminal domain (residues 39-98) is less hydrophilic, such characteristic anomalous migration of E7 proteins in polyacrylamide gel electrophoresis could be expected [25].

Montanide adjuvants have been used for formulation of different protein antigens including long peptides of HPV16 E6 and E7 [26, 27]. The formulations of HPV 16 E6 and E7 peptides with Montanide adjuvants have been used as HPV therapeutic vaccines in clinical trials $[28,29]$. There are limited studies regarding the effect of Montanide ISA 266. The Th2skewed immune responses obtained with our formulation were in agreement with reports indicating that Montanide adjuvants can help eliciting Th2-biased responses [30]. Although the elicited immune responses were mostly Th2-derived, the results of the study showed that the formulated antigens caused the regression of the tumor growth. The distinct role of Th2 cells in tumor eradication in vivo has also been previously shown [31]. Immunization with mutant forms of E7 protein as a safer variant has been previously shown to induce effective antitumor activity [32, 33]. In the present study, both E7 and E7GGG proteins formulated with Montanide ISA 266 elicited moderately effective immune response against TC- 1 induced tumors. In this regard, E7GGG which is considered as a safer alternative, might be a better vaccine candidate to be applied for further studies.

\section{ACKNOWLEDGEMENTS}

We thank Dr. Nasir Mohajel for assistance in statistical analysis, Dr. Mehdi Mahdavi for providing the sequence of the mutated E7 gene sequence and Dr. Azam Bolhasani for providing the sequence of the wild-type E7 gene.

\section{CONFLICT OF INTEREST}

The authors declare that they have no conflict of interest.

\section{REFERENCES}

1. Berman TA, Schiller JT. Human papillomavirus in cervical cancer and oropharyngeal cancer: One cause, two diseases. Cancer. 2017;123(12):2219-29. doi:10.1002/cncr.30588.

2. Kim T, Jin H, Hur S, Yang H, Seo Y, Hong S. Clearance of persistent HPV infection and cervical lesion by therapeutic DNA vaccine in CIN3 patients. Nature communications. 2014; 5: 5317.

3. Hung C-F, Ma B, Monie A, Tsen S-W, Wu T. Therapeutic human papillomavirus vaccines: current clinical trials and future directions. Expert opinion on biological therapy. 2008;8(4):421-39.

4. Frazer IH. Prevention of cervical cancer through papillomavirus vaccination. Nature Reviews Immunology. 2004;4(1):46-55.

5. Ma B, Maraj B, Tran NP, Knoff J, Chen A, Alvarez RD et al. Emerging human papillomavirus vaccines. Expert opinion on emerging drugs. 2012;17(4):469-92.

6. Sun Y, Peng S, Qiu J, Miao J, Yang B, Jeang J et al. Intravaginal HPV DNA vaccination with electroporation induces local CD8\&plus; T-cell immune responses and antitumor effects against cervicovaginal tumors. Gene therapy. 2015;22(7):528-35.

7. Peng S, Wang JW, Karanam B, Wang C, Huh WK, Alvarez RD et al. Sequential cisplatin therapy and vaccination with HPV16 E6E7L2 fusion protein in saponin adjuvant GPI-0100 for the treatment of a model HPV16+ cancer. PloS one. 2015;10(1):e116389.

8. Hallez S, Simon P, Maudoux F, Doyen J, Noël J-C, Beliard A et al. Phase I/II trial of immunogenicity of a human papillomavirus (HPV) type $16 \mathrm{E} 7$ protein-based vaccine in women with oncogenic HPV-positive cervical intraepithelial neoplasia. Cancer Immunology, Immunotherapy. 2004;53(7):642-50.

9. Ressing ME, Van Driel WJ, Brandt RM, Kenter GG, De Jong JH, Bauknecht $\mathrm{T}$ et al. Detection of $\mathrm{T}$ helper responses, but not of human papillomavirus-specific cytotoxic $\mathrm{T}$ lymphocyte responses, after peptide vaccination of patients with cervical carcinoma. Journal of immunotherapy. 2000;23(2):255-66.

10. Borysiewicz L, Fiander A, Nimako M, Man S, Wilkinson GWG, Westmoreland D et al. A recombinant vaccinia virus encoding human papillomavirus types 16 and 18, E6 and E7 proteins as immunotherapy for cervical cancer. The Lancet. 1996;347(9014):1523-7.

11. Kaufmann AM, Stern PL, Rankin EM, Sommer H, Nuessler V, Schneider A et al. Safety and immunogenicity of TA-HPV, a recombinant vaccinia virus expressing modified human papillomavirus (HPV)-16 and 
HPV-18 E6 and E7 genes, in women with progressive cervical cancer. Clinical Cancer Research. 2002;8(12):3676-85.

12. Santin AD, Bellone S, Palmieri M, Ravaggi A, Romani C, Tassi R et al. HPV16/18 E7-pulsed dendritic cell vaccination in cervical cancer patients with recurrent disease refractory to standard treatment modalities. Gynecologic oncology. 2006;100(3):469-78.

13. Smahel M, Polakova I, Sobotkova E, Vajdova E. Systemic administration of $\mathrm{CpG}$ oligodeoxynucleotide and levamisole as adjuvants for gene-gun-delivered antitumor DNA vaccines. Clinical \& developmental immunology. 2011;2011:176759. doi:10.1155/2011/176759.

14. Perrie Y, Mohammed AR, Kirby DJ, McNeil SE, Bramwell VW. Vaccine adjuvant systems: enhancing the efficacy of sub-unit protein antigens. International journal of pharmaceutics. 2008;364(2):272-80. doi:10.1016/j.ijpharm.2008.04.036.

15. Demurtas OC, Massa S, Ferrante P, Venuti A, Franconi R, Giuliano G. A Chlamydomonas-derived Human Papillomavirus 16 E7 vaccine induces specific tumor protection. PloS one. 2013;8(4):e61473.

16. Van de Wall S, W Nijman H, Daemen T. HPV-specific immunotherapy: Key role for immunomodulators. Anti-Cancer Agents in Medicinal Chemistry (Formerly Current Medicinal Chemistry-Anti-Cancer Agents). 2014;14(2):265-79.

17. Stewart-Tull DE. Adjuvant formulations for experimental vaccines. Methods in molecular medicine. 2003;87:175-94. doi:10.1385/1-59259399-2:175.

18. Reyes LE, Gnzález J, Benavides J, García Pariente C, Fuertes M, García Marín JFY et al. New adjuvants in vaccination against ovine paratuberculosis Pathologia. 2002:758- 61.

19. Olson BJ, Markwell J. Assays for determination of protein concentration. Current protocols in protein science. 2007; Chapter 3:Unit 3.4. doi:10.1002/0471140864.ps0304s48.

20. Hallez S, Brulet JM, Vandooren C, Maudoux F, Thomas S, Heinderickx $\mathrm{M}$ et al. Pre-clinical immunogenicity and anti-tumour efficacy of a deleted recombinant human papillomavirus type 16 E7 protein. Anticancer research. 2004;24(4):2265-75.

21. Welters MJ, Filippov DV, van den Eeden SJ, Franken KL, Nouta J, Valentijn AR et al. Chemically synthesized protein as tumour-specific vaccine: immunogenicity and efficacy of synthetic HPV16 E7 in the TC-1 mouse tumour model. Vaccine. 2004;23(3):305-11. doi:10.1016/j.vaccine.2004.06.008.

22. Li YL, Qiu XH, Shen C, Liu JN, Zhang J. Vaccination of full-length HPV16 E6 or E7 protein inhibits the growth of HPV16 associated tumors. Oncology reports. 2010;24(5):1323-9.
23. Watanabe S, Kanda T, Sato $H$, Furuno A, Yoshiike $K$. Mutational analysis of human papillomavirus type 16 E7 functions. Journal of virology. 1990;64(1):207-14.

24. Smahel M, Sima P, Ludvikova V, Vonka V. Modified HPV16 E7 Genes as DNA Vaccine against E7-Containing Oncogenic Cells. Virology. 2001;281(2):231-8. doi:10.1006/viro.2000.0794.

25. Armstrong DJ, Roman A. The anomalous electrophoretic behavior of the human papillomavirus type $16 \mathrm{E} 7$ protein is due to the high content of acidic amino acid residues. Biochemical and biophysical research communications. 1993;192(3):1380-7. doi:10.1006/bbrc.1993.1569.

26. Kenter GG, Welters MJ, Valentijn AR, Lowik MJ, Berends-van der Meer DM, Vloon AP et al. Phase I immunotherapeutic trial with long peptides spanning the E6 and E7 sequences of high-risk human papillomavirus 16 in end-stage cervical cancer patients shows low toxicity and robust immunogenicity. Clinical cancer research : an official journal of the American Association for Cancer Research. 2008;14(1):169-77. doi:10.1158/1078-0432.ccr-07-1881.

27. Aldous AR, Dong JZ. Personalized neoantigen vaccines: A new approach to cancer immunotherapy. Bioorganic \& medicinal chemistry. 2017. doi:10.1016/j.bmc.2017.10.021.

28. Skeate JG, Woodham AW, Einstein MH, Da Silva DM, Kast WM. Current therapeutic vaccination and immunotherapy strategies for HPVrelated diseases. Human vaccines \& immunotherapeutics. 2016;12(6):141829. doi:10.1080/21645515.2015.1136039.

29. Yang A, Farmer E, Wu TC, Hung CF. Perspectives for therapeutic HPV vaccine development. Journal of biomedical science. 2016;23(1):75

30. Coffman RL, Sher A, Seder RA. Vaccine adjuvants: putting innate immunity to work. Immunity. 2010;33(4):492-503. doi:10.1016/j.immuni.2010.10.002.

31. Nishimura T, Iwakabe K, Sekimoto M, Ohmi Y, Yahata T, Nakui M et al. Distinct role of antigen-specific T helper type 1 (Th1) and Th2 cells in tumor eradication in vivo. The Journal of experimental medicine. 1999;190(5):617-27.

32. Grasso F, Negri DR, Mochi S, Rossi A, Cesolini A, Giovannelli A et al. Successful therapeutic vaccination with integrase defective lentiviral vector expressing nononcogenic human papillomavirus E7 protein. International journal of cancer. 2013;132(2):335-44. doi:10.1002/ijc.27676.

33. Li YL, Ma ZL, Zhao Y, Zhang J. Immunization with mutant HPV16 E7 protein inhibits the growth of TC-1 cells in tumor-bearing mice. Oncology letters. 2015;9(4):1851-6. doi:10.3892/ol.2015.2911. 